\title{
The Effect of Citalopram Pretreatment on Neuronal Responses to Neuropsychological Tasks in Normal Volunteers: An fMRI Study
}

\author{
Cristina M Del-Ben', JF William Deakin ${ }^{2}$, Shane Mckie $^{2}$, Nicola A Delvai ${ }^{2}$, Steve R Williams ${ }^{3}$, \\ Rebecca Elliott ${ }^{2}$, Mairead Dolan ${ }^{2}$ and lan M Anderson*,2 \\ 'Faculty of Medicine of Ribeirão Preto, University of São Paulo, Ribeirão Preto, Brazil; ${ }^{2}$ Neuroscience and Psychiatry Unit, The University \\ of Manchester, Manchester, UK; ${ }^{3}$ Imaging Science and Biomedical Engineering, The University of Manchester, Manchester, UK
}

\begin{abstract}
Changes in serotonin neurotransmission have also been implicated in the etiology and treatment of impulse control disorders, depression, and anxiety. We have investigated the effect of enhancing serotonin function on fundamental brain processes that we have proposed are abnormal in these disorders. In all, 12 male volunteers received citalopram (7.5 mg intravenously) and placebo pretreatment in a single-blind crossover design before undertaking Go/No-go, Loss/No-loss, and covert (aversive) face emotion recognition tasks during functional magnetic resonance imaging ( $\mathrm{fMRI}$ ). Blood oxygenation level dependent responses were analyzed using Statistical Parametric Mapping (SPM2). The tasks activated prefrontal and subcortical regions generally consistent with literature with lateral orbitofrontal cortex (BA47) common to the three tasks. Citalopram pretreatment enhanced the right BA47 responses to the No-go condition, but attenuated this response to aversive faces. Attenuations were seen following citalopram in the medial orbitofrontal (BAII) responses to the No-go and No-loss (ie relative reward compared with Loss) conditions. The right amygdala response to aversive faces was attenuated by citalopram. These results support the involvement of serotonin in modulating basic processes involved in psychiatric disorders but argue for a process-specific, rather than general effect. The technique of combining drug challenge with $\mathrm{fMR}$ (pharmacoMRI) has promise for investigating human psychiatric disorders.

Neuropsychopharmacology (2005) 30, 1724- 1734. doi:I0.1038/s..npp. I 300728; published online 13 April 2005
\end{abstract}

Keywords: Go/No-go; face emotion; citalopram; serotonin; fMRI

\section{INTRODUCTION}

Impaired serotonin (5-hydroxytryptamine, 5-HT) neurotransmission has been implicated in the etiology of a wide range of psychiatric disorders including depression, anxiety, and impulse-control-related disorders. Findings common to some or all of these disorders include reduced cerebrospinal fluid concentrations of the 5-HT metabolite, 5 -hydroxyindoleacetic acid, reduced hormonal responses to 5-HT drug challenge, and altered brain 5-HT receptor numbers (Mann et al, 1995; O'Keane et al, 1992; Coccaro et al, 1995; Dolan et al, 2001; Drevets et al, 1999; Stockmeier, 2003). In addition, selective serotonin reuptake inhibitors (SSRIs) have proved effective in their treatment,

*Correspondence: Dr IM Anderson, Neuroscience and Psychiatry Unit, The University of Manchester, Room G907, Stopford Building, Oxford Road, Manchester MI3 9PT, UK, Tel: + 44 I6I 275 7428, Fax: + 44 I6I 275 7429, E-mail: ian.anderson@manchester.ac.uk

Received 13 September 2004; revised 14 February 2005; accepted 16 February 2005

Online publication: 2 March 2005 at http://www.acnp.org/citations/ Npp030205040424/default.pdf an action that is believed to be due to the enhancement of 5-HT neurotransmission (Bell and Nutt, 1998; Mann et al, 2001; Jetty et al, 2001; Nemeroff, 2002).

We, and others, have proposed that alterations in fundamental processes underlying the brain's response to the inhibition of behavior, reinforcement (reward and punishment), and environmental threats could be associated with the development of impulsivity, depression, and anxiety disorders (Deakin and Graeff, 1991; Elliott et al, 2000a). An understanding of the neural basis of these normal neuropsychological processes could prove a useful tool for investigating the abnormal processes involved in psychiatric disorders. Advances in neuroimaging techniques have added substantially to the understanding of neurobiology of emotional processing in humans. Using functional magnetic resonance imaging (fMRI) and positron emission tomography (PET) in conjunction with cognitive activation paradigms, it has been possible to visualize the functional neuroanatomy of specific components of emotional processing such as response to reward and punishment (Elliott et al, 2000b), fear conditioning (Morris et al, 2001), emotion 
recognition (for a review, see Phan et al, 2002), and impulse control (Casey et al, 1997). Cognitive activation paradigms combined with neuroimaging also offer the opportunity to investigate the role of $5-\mathrm{HT}$ in these processes by studying the modulation of neural responses by drugs (pharmacoMRI or pMRI).

Behavioral inhibition of ongoing behavior is an appropriate response to 'stop' signals including aversive stimuli. The inability to inhibit appropriately behavioral responses is a component of impulsivity, which also includes impaired capacity to plan ahead and rapid information processing. In a recent study, we have shown that patients with the diagnosis of borderline and antisocial personality disorder (which is associated with lack of impulse control) activate more widespread prefrontal and temporal cortical areas than healthy controls in the Go/No-go task (Völlm et al, 2004), a neuropsychological task requiring behavioral inhibition (Casey et al, 1997). The Go/No-go task has been widely used as a cognitive activation paradigm with normal volunteers, and it produces consistent prefrontal activation (Horn et al, 2003; Völlm et al, 2004; Rubia et al, 2003; Anderson et al, 2002; Menon et al, 2001; Liddle et al, 2001; Kawashima et al, 1996). The lateral orbitofrontal cortex (OFC), particularly on the right, is a common area activated by No-go compared with Go conditions in these studies and appears to be specifically involved in the inhibition of responses (Aron et al, 2003; Rubia et al, 2003; Menon et al, 2001; Liddle et al, 2001). The right middle frontal gyrus, including dorsolateral prefrontal cortex (DLPFC), also appears to be an important component of behavioral inhibition circuitry (Menon et al, 2001; Garavan et al, 2002). The error detection and processing involved in Nogo performance has been associated with the activation of more medial areas of the prefrontal cortex and the anterior cingulate cortex (Rubia et al, 2003; Garavan et al, 2002; Liddle et al, 2001; Menon et al, 2001; Hester et al, 2004). We have previously shown enhanced neuronal responses in the lateral OFC to Go/No-go performance after the administration of $m$-chlorophenypiperazine (mCPP), a $5-\mathrm{HT}_{2 \mathrm{C}}$ agonist (Anderson et al, 2002), consistent with a direct modulatory effect of 5-HT on the neural circuits involved in behavioral inhibition.

From an evolutionary perspective, it has been proposed that the neural substrates involved in the behavioral response to acute and chronic stress, punishment and threat, could be implicated in the neurobiology of anxiety and depressive disorders, with a central role for 5-HT in the modulation of these mental processes (Deakin and Graeff, 1991; Deakin, 2003). We have previously shown that a simple financial reward task activates the amygdala and striatum (Elliott et al, 2003), while financial loss is associated with increased neuronal response in the hippocampus (Elliott et al, 2000b). The OFC has also been implicated in recognition and processing of reward and punishment stimuli, with some findings suggesting an anatomical division of its functions with lateral areas associated with loss/punishment and medial areas more with reward (Bechara et al, 1994; O'Doherty et al, 2001; Elliott et al, 2003). It has been argued that lateral regions of the OFC are critical in responding to punishments signalling a need for behavioral change (Kringelbach and Rolls, 2004). Depressed patients show abnormal
OFC responses to financial rewards and losses (Longe et al, 2003). Previous work in our unit has shown that reducing brain 5-HT function using acute tryptophan depletion increases depressive and anxiety reactions to laboratory stress (Miller et al, 2000; Anderson et al, 2003), and there is preliminary evidence showing 5-HT modulation of the processing of reward and punishment cues involved in decision making (Rogers et al, 2003). Little, however, is known about the functional neuroanatomy of 5-HT involvement in basic processes of reinforcement in humans.

The ability to identify facial expressions and emotions is an important component of social functioning and adaptation. There is evidence for an involvement of dissociable, although interconnected, neural substrates that mediate face emotion processing, with a central role for the amygdala (Haxby et al, 2002). The amygdala has been one of the most consistently activated areas in studies relating to fearful faces (eg Morris et al, 1996; Hariri et al, 2000; Phillips et al, 2001), a response that is lateralized in some studies (eg Morris et al, 1996), possibly relating to different rates of habituation with one study finding faster habituation in the right than left amygdala to repeated exposure (Morris et al, 2001). There are less consistent findings with other emotions such as anger (eg Blair et al, 1999; Hariri et al, 2002) and disgust (Phillips et al, 1997; Winston et al, 2003), perhaps reflecting a stronger reaction to fear than other emotions rather than a truly selective response (Whalen et al, 2001). Previous imaging studies using emotional face stimuli have also shown activation of medial and orbital prefrontal cortices, including anterior cingulate, insula and regions of the occipital cortex, particularly the fusiform gyrus (Sprengelmeyer et al, 1998; Blair et al, 1999; Surguladze et al, 2003; Abel et al, 2003; Keightley et al, 2003). Depressed and anxious patients have been shown to have altered neural responses to negative emotional faces (Lawrence et al, 2004; Thomas et al, 2001; Sheline et al, 2001). Antidepressant treatment has been shown to normalize increased amgydala responses to fearful faces in depressed patients (Sheline et al, 2001; Fu et al, 2004). Acute modulation of 5-HT function alters facial expression recognition in healthy volunteers (Harmer et al, 2003a, b; Attenburrow et al, 2003), but as yet it is unclear as which to neural substrates mediate this effect.

The aim of this study was to determine the acute effects of the SSRI, citalopram, on brain activation during cognitive activation tasks tapping into fundamental neuropsychological processes that could be involved in the pathophysiology of psychiatric diagnosis (behavioral inhibition, reinforcement processing, and covert aversive face emotion recognition).

Specifically, on the basis of the literature reviewed above, we hypothesized that performance of the cognitive tasks would be associated with distinct but overlapping patterns of activation as follows:

1. Go/No-go task: Our specific hypotheses concerned prefrontal cortical regions. We predicted that in line with the previous studies discussed, No-go relative to Go blocks would be associated with increased BOLD response in lateral OFC, anterior cingulate, right middle frontal gyrus, and DLPFC. 
2. Loss task: We hypothesized that the main effect of loss compared to no loss would be associated with neural responses in the lateral OFC and hippocampus.

3. Covert recognition of aversive faces: For this task, we predicted increased BOLD response in the amygdala, fusiform gyrus, OFC, and anterior cingulate gyrus.

A response in the OFC was predicted for all three tasks. This is a region that mediates a variety of social, cognitive, and emotional functions, and, as discussed above, appears to play a critical role in all three of the functions assessed here. For each of the tasks, we further predicted that citalopram would modulate the neuronal responses associated with task performamance. Based on previous results, and the clinical profile of the drug, we hypothesized that citalopram would enhance neuronal activation induced by these tasks. We term this approach to imaging the effects of drugs on cognitive activation as 'indirect' pharmacological fMRI (pMRI) to distinguish it from direct neuronal activation by drugs ('direct' pMRI).

\section{METHODS}

\section{Subjects}

In all, 12 healthy (10 right handed) male volunteers, aged 19-36 years (mean \pm SD $24.7 \pm 5.8$ years) were recruited from students, staff, and the general public, through advertisement.

Subjects were screened using the Overview Module from Structured Clinical Interview for DSM-IV-TR Axis I Disorders, Research Version, Non-patient Edition (SCIDNP; First et al, 2002) and the Mini International Neuropsychiatry Interview (MINI; Sheehan et al, 1998). Exclusion criteria included any serious general medical condition or one that could interfere in the interpretation of results, current or past psychiatric disorder, use of medication within the last 2 weeks, illicit drug use, excessive consumption of alcohol ( $>21 \mathrm{U} /$ week), caffeine ( $>8$ cups of coffee/ day), or cigarette consumption ( $>10$ cigarettes/day), and involvement in concurrent research or in research involving taking an experimental drug in the previous 2 months.

The study was approved by the local Research Ethics Committee and written informed consent was obtained from each volunteer.

\section{Procedures}

Subjects were tested on two occasions with placebo (normal saline) or intravenous citalopram $7.5 \mathrm{mg}$, infused over $7.5 \mathrm{~min}$, separated by at least 3 days (range 3-28 days, mean $\pm S D=11.1 \pm 6.9$ days), in a randomized, balanced order, single-blind design. The choice of citalopram was based on its high selectivity for 5-HT, evidence from animal and human studies for serotonergic effects, its good tolerability, and its availability as an intravenous preparation (see Discussion). Subjects were cannulated outside the scanner at least $30 \mathrm{~min}$ before they received the drug. They then underwent a $22.5 \mathrm{~min}$ fMRI scan during which they received the drug infusion in the middle $7.5 \mathrm{~min}$ in order to examine the direct effects of citalopram. These results are not presented here. At $5 \mathrm{~min}$ after the end of this scan (ie
$12.5 \mathrm{~min}$ after the end of the drug infusion), they received the first of three further scans during which they undertook the neuropsychological tasks detailed below.

Neuropsychological Tasks. Three tasks of a block-design lasting $6 \mathrm{~min}$ each were performed inside the scanner in the order Go/No-go, Loss/No-loss, and covert face emotion recognition.

Go/No-go. This task was based on one reported by Casey et al (1997) and previously used in our Unit (Horn et al, 2003; Anderson et al, 2002). Subjects were presented with a series of letters displayed for $500 \mathrm{~ms}$ every $1.73 \mathrm{~s}$ on a screen and instructed to respond, using a button box, to all letters apart from ' $\mathrm{V}$ ' by pressing a button. Eight blocks, of $45 \mathrm{~s}$ each, were presented in an $A B A B A B A B$ design, $A$ being the Go condition, with no V's and B the No-go condition, with $50 \%$ V's.

Loss/No-loss. This task consisted of six blocks, three associated with loss (2nd, 4th and 6th) and three with no loss (1st, 3rd, and 5th). Subjects were presented with a series of colored squares at a rate of one every $1.33 \mathrm{~s}$. They were instructed to respond only to blue and green squares by pressing a button as fast as they could. Volunteers were instructed that if they were not quick enough to respond on blue squares, they would lose money but they would not lose money on green squares. Losses occurred for $65 \%$ of the blue targets and were signalled by pound signs with a cross through them.

Covert Face Emotion Recognition. Subjects were presented with pictures of faces from the Pictures of Facial Affect Series (Ekman and Friesen, 1976). The following types of affect were presented: neutral (A), anger (B), disgust (C), and fear (D). Eight pictures of each emotion was presented for $3 \mathrm{~s}$ with an interval of $0.75 \mathrm{~s}$ between presentations. Each block lasted $30 \mathrm{~s}$, presented in an ABACADABACAD design. Using a button box, subjects were asked to identify the gender of the faces not to describe or focus on the emotion pictured.

fMRI Scanning. Images were acquired using a Philips (Eindhoven, Holland) $1.5 \mathrm{~T}$ Gyroscan ACS NT retrofitted with Powertrak 6000 gradients, operating at a software level 6.1.2 $\mathrm{T} 2{ }^{\star}$-weighted volumes were acquired using a singleshot echo-planar (EPI) pulse sequence. Each volume comprised 40 contiguous axial slices, $(\mathrm{TR} / \mathrm{TE}=5000 /$ $40 \mathrm{~ms}, 64 \times 64$ data matrix, $3.5 \mathrm{~mm}$ thickness with an inplane resolution of $3 \times 3 \mathrm{~mm}$ ). A T1-weighted structural scan was also acquired for each subject, for coregistration and to exclude any structural abnormality. No abnormality was reported for any of the 12 subjects.

Analysis. Imaging data were analyzed using Statistical Parametric Mapping (SPM2, Friston, The Welcome Department of Cognitive Neurology, London, UK), with a random effects model. Images were realigned to correct for motion artifacts using the first scan as a reference and normalized into the Talairach and Tournoux stereotactic space (Talairach and Tournoux, 1988) using Montreal Neurological Institute (MNI) templates. Images were smoothed 
with a $10 \mathrm{~mm}$ Gaussian kernel to facilitate intersubject averaging. After this spatial preprocessing, first-level analysis was performed on each subject using the general linear model with a delayed boxcar waveform to model blood oxygenation level-dependent (BOLD) signal changes to generate a single mean image corresponding to each experiment. These images were combined in a second-level, random effects, analysis, using a one-way ANOVA to investigate the main effect of the task. This main effect was used as an inclusive mask to examine which of the regions responsive to the task showed a significant modulation by the drug relative to placebo (ie an interaction between task and drug, masked by the main effect of task). It is possible for significant interactions to occur outside areas where a main effect is seen, but it is then difficult to interpret their functional significance.

For the three tasks, neural responses in the control blocks were subtracted from those in the active task blocks, to reveal areas of increased signal associated with performance of the task, irrespective of drug pretreatment. The active minus control comparisons were No-Go minus Go, Loss minus No-loss, and aversive emotion minus neutral in the three tasks, respectively. In the Loss/No-loss task, the reverse subtraction was also examined. In the analysis of the covert face emotion recognition task, the combined aversive faces analysis compared with neutral faces is presented in order to identify brain areas responding to external threat in a generic sense rather than specific emotions. Within areas showing a main effect of task, neural responses on placebo were subtracted from those on citalopram to identify areas where citalopram enhanced neural activation to the task, with the reverse subtraction used to identify where citalopram attenuated responses. Statistical maps were thresholded at $p<0.001$ uncorrected $(Z$-score $>3.09)$ with only cluster sizes of 10 or more contiguous voxels being reported. Small volume corrections (Worsley et al, 1996) were applied to a priori regions of interest, as specified in the introduction. These regions, and the volume of correction, were chosen on the basis of previous studies reported by ourselves and others (eg Morris et al, 1996; Hariri et al, 2000; Phillips et al, 2001; Elliott et al, 2003; Horn et al, 2003). In addition, lower levels of significance are reported when bilateral activations were seen. We also report regions where activation was observed significant at $p<0.001$ uncorrected. However, these regions are included for descriptive purposes only and are not discussed further.

\section{RESULTS}

Citalopram administration was very well tolerated and there were no significant differences between visual analog selfratings of mood, discomfort, nausea, and sedation/alertness taken before and after the scanner tasks. Behavioral results for one subject were not available because of a technical problem.

\section{Go/No-go Task}

There were no differences between occasion in the number of omission errors (not responding to letters apart from V; mean \pm SD: $0.3 \pm 0.5 \%$ for placebo, $0.2 \pm 0.8 \%$ for citalopram, $p=0.9$ ) or commission errors (responding to V's; $11.2 \pm 4.8 \%$ for placebo, $10.7 \pm 6.6 \%$ for citalopram, $p=0.7$ ). Subjects were slightly slower to respond on the citalopram occasion (reaction time on placebo $387 \pm 28 \mathrm{~ms}, 406 \pm 24 \mathrm{~ms}$ on citalopram, $p=0.05$ ).

The main effects of task and their modulation by citalopram are represented in Table 1 and Figure 1. During the No-go blocks (behavioral inhibition) significant neuronal responses after small volume correction on the basis of prior hypothesis were observed in the right DLPFC, OFC bilaterally, and right middle frontal gyrus. Additional responses, significant at $p<0.001$ uncorrected, were observed in right temporal cortex, bilateral supramarginal gyri, right precuneus, and right thalamus. Citalopram enhanced the activation of DLPFC, right lateral OFC, and middle temporal gyrus. Attenuations were seen in OFC, more on the left, bilateral supramarginal gyri, and right superior temporal gyrus.

\section{Loss/No-loss Task}

Subjects performed near ceiling on this task with very few commission or omission errors and no difference in reaction time between the two occasions (data not shown).

As shown in Table 2, the main effects of the Loss compared with No-loss blocks in prehypothesised regions were observed bilaterally in lateral OFC. Additional responses significant at $p<0.001$ uncorrrected were observed in bilateral frontopolar prefrontal cortex, and parietal cortex, predominantly left temporal cortex and right fusiform gyrus. Citalopram decreased the response in right fusiform gyrus (Table 2).

In the No-loss compared with Loss blocks, increased hemodynamic responses were seen in bilateral medial OFC, right anterior cingulate gyrus, right insula, mainly left temporal cortex, left occipital cortex, predominantly right parahippocampal gyrus, right hippocampus, and uncus. Citalopram increased the activation in right insula and left occipital cortex. The activation of right medial OFC was attenuated by citalopram (Table 2, Figure 1c).

\section{Covert Face Emotion Recognition}

Subjects performed near ceiling on this task with very few incorrect responses or omission errors and no difference in reaction time between the two occasions (data not shown).

Table 3 shows that aversive faces, when compared with neutral faces, caused bilateral neuronal activation in hypothesized regions of lateral OFC, fusiform gyri, and amygdala/amygdaloid complex. Additional responses, significant at $p<0.001$ uncorrected, were observed in DLPFC, superior temporal cortices, occipital gyri, and thalami. Citalopram tended to enhance the activation in fusiform gyri and thalami bilaterally, whereas it attenuated the activation in the right lateral OFC and right amygdala (Figure 1d).

\section{DISCUSSION}

We have studied regional neuronal activation, and its modulation by $5-\mathrm{HT}$, in three paradigms selected to probe 
Table I Maximally Activated Voxels in Areas in which Significant Evoked Activity was Related to No-go Block Compared to Go Block Levels

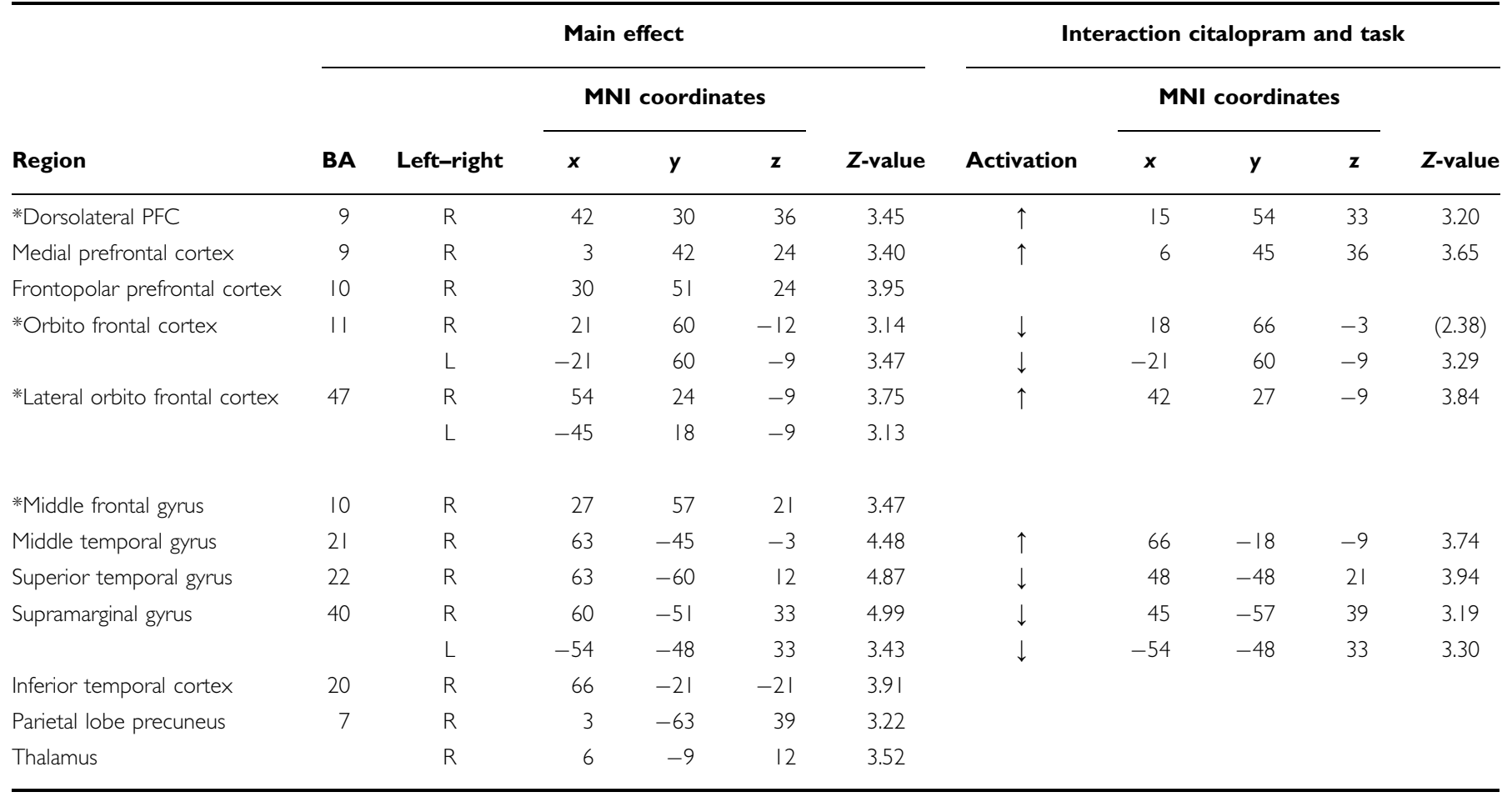

Statistical maps were thresholded at $p<0.001$ uncorrected. regions marked * are those regions about which we had a prior hypothesis based on previous imaging studies using this task.

BA $=$ Brodmann Area; $\downarrow=$ attenuated the activation; $\uparrow=$ enhanced the activation; figures in brackets are below threshold but included because the modulation appears bilateral.
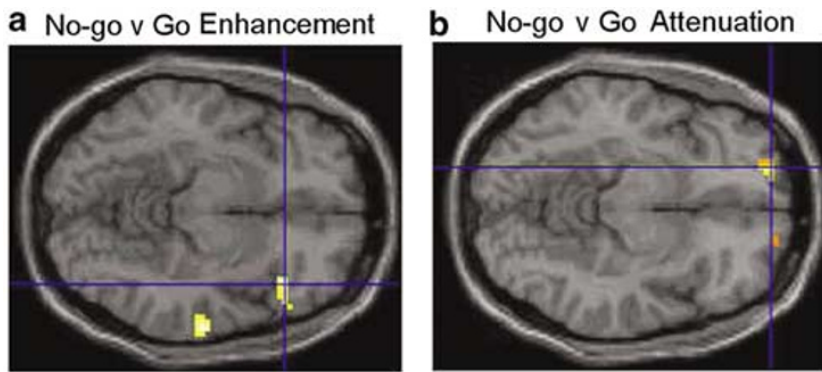

C

No-loss v Loss Attenuation

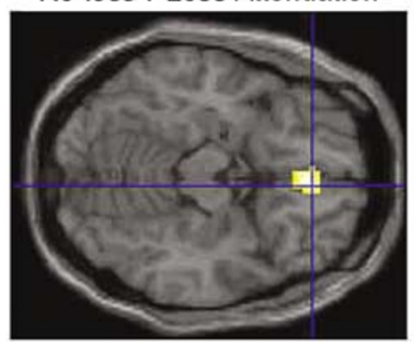

d Aversive $v$ Neutral faces

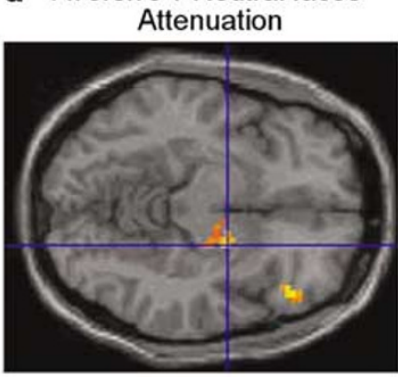

Figure I Modulation of task-related activations by citalopram. (a) Enhancement of right BA47 activation in No-go compared with Go blocks. (b) Attenuation of left > right BAI I activation in No-go compared with Go blocks. (c) Attenuation of right BAII activation in No-loss compared with Loss blocks. (d) Attenuation of right BA47 and right amygdala activation in Aversive compared with Neutral face blocks. neuropsychological processes related to impulsivity, depression, and anxiety, namely behavioral inhibition, loss, and viewing of aversive faces. The main effects of the tasks in this study (ie independent of drug modulation) have confirmed the involvement of prefrontal and subcortical regions, which in general are consistent with other findings in the literature (Casey et al, 1997; Rubia et al, 2003; Horn et al, 2003; Haxby et al, 2002). Acute infusion of the SSRI, citalopram, modulated brain responses in a task-specific manner with only modest overlap. This supports a role for 5-HT in these process, but one that is crucially dependent on the nature of the psychological task involved.

\section{Go/No-go Task}

Some studies have shown impaired performance in this task related to poor impulse control (Dolan and Park, 2002), although some negative results have also been reported (Dinn and Harris, 2000). Given the inverse relationship that has been found between 5-HT function and impulsivity (eg Dolan et al, 2001), it is of interest that subjects tended to respond to targets more slowly under citalopram; however, there was no effect on the number of commission errors. We found that citalopram enhanced right OFC activation during No-go blocks, a similar finding to our previous result with the $5-\mathrm{HT}_{2 \mathrm{C}}$ agonist, $\mathrm{mCPP}$ (Anderson et al, 2002), supporting a modulatory role for 5-HT in this region, 
Table 2 Maximally Activated Voxels in Areas in which Significant Evoked Activity was Related to Loss and No-Loss Blocks Levels

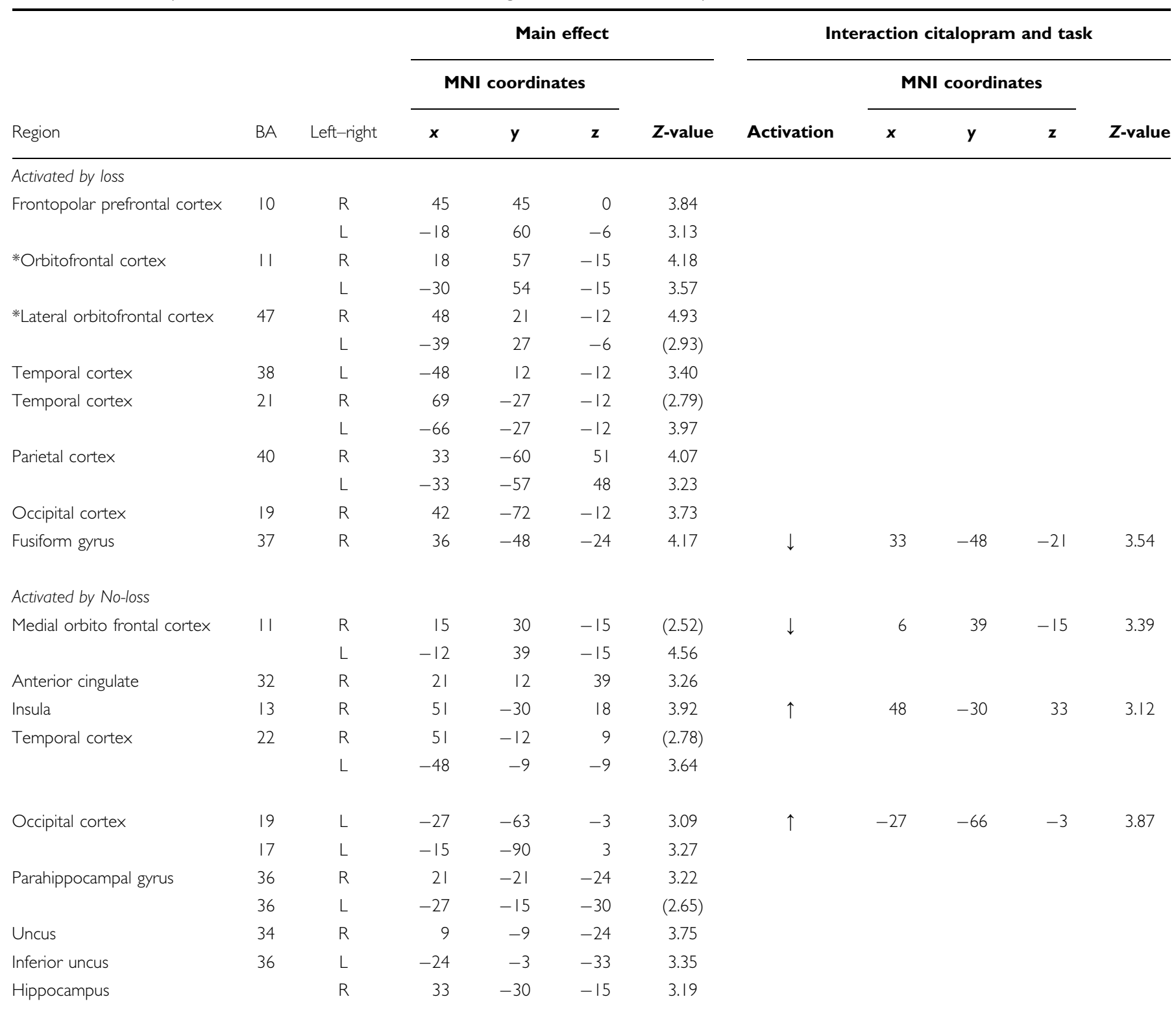

Statistical maps were thresholded at $p<0.001$ uncorrected. Regions marked * are those regions about which we had a prior hypothesis based on previous imaging studies using this task.

$\mathrm{BA}=$ Brodmann Area; $\downarrow=$ attenuated the activation; $\uparrow=$ enhanced the activation; figures in brackets are below threshold but included because the activation appears bilateral.

probably $5-\mathrm{HT}_{2 \mathrm{C}}$ receptor mediated. Citalopram also increased activation of other right-sided areas including DLPFC, an area that has been implicated in working memory load in this task (Mostofsky et al, 2003). In contrast citalopram attenuated more medial OFC and superior temporal cortex/supramarginal gyrus. These findings are consistent with a role for 5-HT in the effective inhibition of unwanted behaviors and suggest a direct involvement in the circuitry involved in withholding responses. The attenuation by citalopram of activation in areas that may be involved in error processing and attention/spatial awareness (Karnath, 2001) suggest a different role for 5-HT in this circuitry.

\section{Loss/No-loss Task}

Understanding the results obtained in the Loss/No-loss task is complicated by features of the task itself that became apparent after the study. Although the No-loss blocks were designed as neutral blocks, given the subtraction design in relation to the Loss bocks, they could be perceived as associated with not losing, or relative reward. In addition, within the Loss Block, there was an expectation of losing for about $\frac{2}{3}$ of the time, and also the experience of avoiding loss for the rest of the time. Learning theory has long regarded omission of anticipated punishment as functionally equivalent to reward. Indeed, subjects might have consciously 
Table 3 Maximally Activated Voxels in Areas in which Significant Evoked Activity was Related to the Covert Recognition of Aversive Faces Blocks Compared to the Recognition of Neutral Faces Blocks

\begin{tabular}{|c|c|c|c|c|c|c|c|c|c|c|c|}
\hline \multirow{2}{*}{ Region } & \multicolumn{6}{|c|}{ Main effect } & \multicolumn{5}{|c|}{ Interaction citalopram and task } \\
\hline & BA & Left-right & \multicolumn{3}{|c|}{ MNI coordinates } & Z-value & Activation & \multicolumn{3}{|c|}{ MNI coordinates } & Z-value \\
\hline \multirow[t]{2}{*}{ *Lateral orbitofrontal cortex } & 47 & $\mathrm{R}$ & 48 & 30 & -9 & $(3.05)$ & $\downarrow$ & 45 & 28 & -15 & 4.18 \\
\hline & & L & -30 & 18 & -24 & 3.15 & & & & & \\
\hline & & L & -42 & 33 & 15 & 3.23 & & & & & \\
\hline & 9 & $R$ & 18 & 45 & 39 & 3.59 & & & & & \\
\hline \multirow[t]{2}{*}{ Temporal cortex } & 38 & R & 39 & 12 & -42 & 4.29 & & & & & \\
\hline & & L & -39 & 15 & -42 & 3.63 & & & & & \\
\hline Occipital cortex & 18 & R & 39 & -90 & -9 & 5.35 & & & & & \\
\hline *Amygdaloid complex & 34 & $\mathrm{R}$ & 15 & -6 & -12 & 3.73 & $\downarrow$ & 21 & -3 & -9 & $2.50 *$ \\
\hline *Amygdala & & L & -24 & -12 & -15 & 3.26 & & & & & \\
\hline \multirow[t]{2}{*}{ Thalamus } & & $R$ & 9 & -21 & 6 & $(3.04)$ & $\uparrow$ & 3 & -30 & 0 & $(3.01)$ \\
\hline & & L & -18 & -21 & 9 & 3.42 & $\uparrow$ & -9 & -27 & 0 & $(2.23)$ \\
\hline
\end{tabular}

Statistical maps were thresholded at $p<0.00$ I uncorrected. Regions marked * are those regions about which we had a prior hypothesis based on previous imaging studies using this task.

$\mathrm{BA}=$ Brodmann Area; $\downarrow=$ attenuated the activation; $\uparrow=$ enhanced the activation; * small volume correction $p<0.05$ (radius of voxels of interest $=5 \mathrm{~mm}$ ); figures in brackets are below threshold but included because the activation/modulation appears bilateral.

experienced omission of loss as mildly relieving or as a pleasurable consequence of their own quick reactions.

Given the caveats and this interpretation of the No-loss condition, the areas showing main effects of task are compatible with areas identified in the literature. As predicted by previous studies of financial loss (eg O'Doherty et al, 2001), activation of lateral areas of OFC was more pronounced during the Loss blocks than during the No-loss blocks. The opposite subtraction showed medial OFC and right anterior cingulate activation. This was not a planned comparison and therefore we did not have a prior hypothesis concerning this effect. However, the findings are consistent with what we would have predicted based on the interpretation that No-loss is relative rewarding compared with Loss. O'Doherty et al (2001) found activation of the medial OFC to financial reward. Other studies have also reported medial OFC responses to rewarding stimuli (see Kringelbach and Rolls, 2004, for a review).

In a previous study, involving financial loss in a choice paradigm, Elliott et al (2000b) observed bilateral hippocampal activation and we expected to see the same. However, we observed no hippocampal activation; indeed, right hippocampus was activated in the No-loss condition. The explanation is not obvious, but may relate to different contexts of the tasks.

Citalopram attenuated response to loss in right fusiform gyrus (see above). A possible implication is that 5-HT decreases attention to, or processing of, visual stimuli that predict an aversive outcome that cannot be avoided. Some enhancement of the right OFC response was observed, as in the No-go task, but this fell short of statistical significance $(z=2.38)$. Our expectation had been that citalopram might modulate prefrontal and temporal effects of loss given the experimental evidence for a role of 5-HT in anxiety and responses to punishment (Graeff, 2002) as well as clinical relevance in treating mood and anxiety disorders (Bell and Nutt, 1998; Anderson, 2001). It is possible that the loss aspect of this task was insufficiently potent or relevant to individuals to engage circuits involved in emotion, or that the task itself was confounded as discussed above.

Citalopram attenuated right medial OFC activations in the No-loss blocks suggesting a possible modulation of circuits involved in reward. Citalopram enhanced occipital activation, which is consistent with 5-HT enhancement of earlystage visual information processing as suggested by a previous finding that fenfluramine increased critical flicker fusion threshold (Andrews and Anderson, 1998).

\section{Covert Face Emotion Recognition}

Since first described by Darwin (1872), facial expressions of basic emotions have been considered a key component of emotion processing. A considerable number of imaging studies involving pictures of face emotion have been conducted with evidence to support the idea that different emotions processes involve separate neural systems. 
Nevertheless, there are also findings suggesting a common pathway to facial expression processing, involving amygdala and prefrontal areas, especially the lateral OFC. We do not report separate analyses for different negative expressions in this paper, but concentrate on the common effects of negative facial emotions since we have speculated that $5-\mathrm{HT}$ modulates responses to aversion.

Consistent with others' findings we found activation of the parahippocampal gyrus bilaterally extending into the amygdala in response to aversive faces. Amygdala activation has been reported in a number of studies to be activated by a range of emotional faces (Winston et al, 2003; Yang et al, 2002) and by novel compared with familiar faces (Schwartz et al, 2003), suggesting that the amygdala may be activated by stimuli of potential significance or threat to the individual.

Aversive faces also activated posterior OFC on the right, lateral frontal cortex, and temporal poles on both sides, occipital and extrastriate regions, particularly fusiform gyrus, again broadly consistent with other studies (Keightley et al, 2003; Lange et al, 2003; Gur et al, 2002; Abel et al, 2003; Surguladze et al, 2003; Sprengelmeyer et al, 1998).

Acute citalopram decreased activation in the right lateral OFC and right parahippocampal/amygdala region (with small volume correction). Increases in fusiform gyri and thalamic responses were bilateral, but individually below the threshold for significance.

Behavioral studies with acute citalopram and tryptophan depletion and supplementation (Harmer et al, 2003a,b; Attenburrow et al, 2003) report increased recognition of fearful faces associated with increased 5-HT function, in women. Subchronic citalopram administration in contrast was associated with decreased recognition of fearful faces (Harmer et al, 2004). These findings are consistent with the proposal that acute increases in 5-HT can be associated with increased anxiety (Mortimore and Anderson, 2000; Hetem et al, 1996), and that decreased anxiety and therapeutic efficacy result from adaptive changes in the 5-HT system with continued treatment (Bell and Nutt, 1998). Our findings suggest that 5-HT attenuates the processing of emotion in faces in limbic structures. However, this further implies that the heightened detection of fear in faces induced by citalopram (Harmer et al, 2003a) is not mediated by 5 -HT modulation of amygdala function. Our results suggest it may be due to enhancement of visual processing in fusiform cortex and thalamic attentional mechanisms. However, as discussed above, the equation of activation with functional activity is not straightforward and we did not record performance on face emotion identification. Therefore, we do not know whether our subjects would have behavioral data comparable with Harmer et al (2003a). Indeed, the latter study involved female subjects, whereas the present study was carried out in males. Attenburrow et al (2003), using tryptophan supplementation, found increased sensitivity to recognition of fearful faces in women but not in men, suggesting there may be a differential sex effect. There is also evidence of an inverse relationship between activation in hippocampal/amygdala regions compared with prefrontal areas dependent on the degree of conscious identification of emotion (Hariri et al, 2002; Lange et al, 2003), with conscious labelling increasing prefrontal activation. It is therefore possible that $5-\mathrm{HT}$ is involved in processes related to the bringing of aversive emotions to conscious awareness.

Of interest, a recent study in depressed patients reported increased left hemisphere responses to sad faces compared with controls including amygdalar/parahippocampal area, ventral striatum, caudate, thalamus, and dorsal cingulate gyrus. Treatment for 8 weeks with the SSRI fluoxetine led to a reduction in responses, that did not, however, correlate with symptomatic improvement, although an increase in range of haemodynamic response in the anterior cingulate gyrus was associated with response (Fu et al, 2004). While not directly comparable with our paradigm, these results do support a modulation of amydgala responses to face emotion by 5 -HT.

\section{Comparison of Citalopram Modulation in Different Task-Related Activations}

It is striking that right lateral OFC activations were seen in all three tasks in this experiment. This area receives polymodal sensory inputs and is involved in the lateral orbitofrontal-basal ganglia loop (Zald and Kim, 2001). Our findings are in keeping with the proposal that right BA47 is engaged in situations were a behavioral strategy requires reappraisal and behavioral inhibition because outcomes change or become aversive (Elliott et al, 2000a; Wallis et al, 2001). Acquired lesions of this region in humans interferes with response inhibition in the Stop paradigm, a type of Nogo task (Aron et al, 2003). Only right-sided lesions have this effect and this is compatible with more pronounced rightsided effects in our study and enhancement of right BA47 activation by citalopram, both of which suggest a genuine lateralization of function.

It is interesting that citalopram enhanced this activation in the Go/No-Go task but attenuated the response to aversive faces. We also observed a tendency to enhance the response in the loss task which failed to reach significance. One possible explanation is that 5 -HT promotes activity in this region when a behavioral response is required, such as response inhibition, but when this response selection system is engaged by aversive processes, without behavioral implications, citalopram attenuates the response.

Medial OFC (BA11) activation was seen in both the Go/ No-go tasks and the No-loss condition of the monetary task; however, there was little overlap between the activated voxels. This general region is part of the medial orbitofrontal-basal ganglia loop with connections with the anterior cingulate cortex, ventral striatum, medial temporal structures including the amygdala, and hypothalamus (Zald and Kim, 2001). It appears to be engaged by reinforcing stimuli when there is a stimulus discrimination component to the task, and this may have a functional similarity with error-checking in the Go/No-go task. In both tasks, citalopram attenuated the response, but the implications for the role of 5-HT are not obvious.

We designed our tasks to be very simple to minimize performance differences or effects of difficulty, which could have confounded interpretation of results if citalopram had affected these. We acknowledge that this can also be seen as a limitation of the study as it was not designed to explore to what extent these might explain the drug effects seen, or differences between tasks. While we believe this is unlikely 
in the current study, a challenge for future pMRI studies will be to explore the relationship between drug effects on neuronal activation and neuropsychological aspects such as subjective state, perceived difficulty and performance.

\section{Methodological Considerations}

It is important to consider difficulties inherent in the interpretation of BOLD signal modulation following drug administration. These include the effects of the drug on 5-HT function, nonspecific effects of the drug on BOLD signal, and the interpretation of increased or decreased 'activation' identified by altered BOLD signal.

Microdialysis studies in animal have consistently shown acute increases in cortical extracellular 5-HT following acute SSRI administration (David et al, 2003; Felton et al, 2003; Moret and Briley, 1996). However, animal studies have typically given doses 10-100 times higher than the low-dose (about $0.1 \mathrm{mg} / \mathrm{kg}$ ) we administered (eg Felton et al, 2003). Our dose was determined by considerations of safety and tolerability in acute intravenous infusion because we also wanted to examine the direct neural effects of citalopram (direct pMRI, data not presented here). There is evidence that increases in extracellular 5-HT following SSRIs are greater in the raphe nuclei than the cortex (Bel and Artigas, 1992). It is therefore possible that acute low dose citalopram could preferentially inhibit 5-HT reuptake in cell body, rather than terminal, areas. This would lead to reduced neuronal firing rate through somatodendritic autoreceptor activation (eg Gartside et al, 1995), and hence lower terminal 5-HT release and lower synaptic concentrations. However, two lines of evidence led us to believe that this dose was sufficient to increase terminal synaptic 5-HT concentrations. First, neuroendocrine challenge with intravenous citalopram at the low dose of $5 \mathrm{mg}$ has been shown to result in plasma hormonal responses (Attenburrow et al, 2001), and in this study, we also found significant increases in prolactin and cortisol concentrations (data not presented). Second, the effect of acute oral citalopram on face emotion recognition is opposite to that seen with acute tryptophan depletion and similar to that seen after tryptophan supplementation (Harmer et al, 2003a,b; Attenburrow et al, 2003). Nevertheless, we acknowledge that there remains some uncertainty about the net effect of acute SSRI administration in our study, which must be considered in interpreting the data. It is possible that higher citalopram doses than used here might have resulted in a wider, or different, pattern of modulation. In addition, the effect of postsynaptic 5-HT receptor stimulation is often inhibitory, particularly when $5-\mathrm{HT}_{1 \mathrm{~A}}$ receptors are involved, so it is also difficult to know whether enhanced (or reduced) 5 -HT function will lead to regional neuronal activation or inhibition.

The possibility of nonspecific effects of citalopram also need to be considered (eg increasing blood flow in activated regions), but we would argue that our findings of areas of decreased BOLD signal, and opposite effects in the same region depending on task, argue against this and for a taskspecific modulation by 5-HT. A further question in interpreting pharmacological modulation of neuropsychological tasks using fMRI is what the changes in brain activation mean in terms of the underlying neuronal processes or circuitry. Increases in BOLD signal are believed to be an indirect measure of increased neuronal metabolism, but does an increase reflect neurones being more activated because the task is being performed better, or because more cells have to be recruited to achieve the same level of function? Other uncertainties lie in the interpretation of block design analyses where the comparison is based on a subtraction of BOLD signal in the two conditions. Relative increase in BOLD signal in one type of block compared with another could be due to the activation in that block or attenuation in the comparator block. In addition, block designs are insensitive to, and may be confounded by, changes in response over the time of testing or between blocks. For example, rapid habituation of amygdala activation to faces is well described (Fischer et al, 2003; Thomas et al, 2001) and could lead to activation following initial stimulus presentation not being detected because it is outweighed in the overall analysis by lack of activation subsequently.

Bearing these limitations in mind, we believe that the results do start to build a picture of 5-HT modulation of neuronal circuitry involved in basic psychological processes.

\section{CONCLUSIONS}

We were interested in both similarities and differences in the effect of citalopram on activations in the three tasks, which were chosen to probe aspects of psychological functioning that might underlie areas of psychopathology in which 5-HT is believed to be involved. The tasks did show different patterns of activation, although there was a common theme of OFC involvement. Our results support the involvement of $5-\mathrm{HT}$ in each of these processes, although the activations occurring in Loss/No-loss task are difficult to interpret in a straightforward way. A major conclusion from our study is the task-specific nature of citalopram modulation of neuronal activation. This is consistent with the complexity implied by the widespread innervation by 5-HT neurones and the involvement of 5-HT in a wide variety of different functions.

\section{ACKNOWLEDGEMENTS}

The study was supported by the Medical Research Council (UK) and Dr Del-Ben was supported by FAPESP (Fundação de Amparo à Pesquisa do Estado de São Paulo) and CAPES (Coordenação de Aperfeiçoamento de Pessoal de Nível Superior).

\section{REFERENCES}

Abel KM, Allin MP, Kucharska-Pietura K, David A, Andrew C, Williams $S$ et al (2003). Ketamine alters neural processing of facial emotion recognition in healthy men: an fMRI study. Neuroreport 14: 387-391.

Anderson IM (2001). Meta-analytical studies on new antidepressants. Br Med Bull 57: 161-178.

Anderson IM, Clark L, Elliott R, Kulkarni B, Williams SR, Deakin JF (2002). 5-HT(2C) receptor activation by $m$-chlorophenylpiperazine detected in humans with fMRI. Neuroreport 13: $1547-1551$. 
Anderson IM, Richell RA, Bradshaw CM (2003). The effect of acute tryptophan depletion on probabilistic choice. J Psychopharmacol 17: 3-7.

Andrews TM, Anderson IM (1998). Information processing in anxiety: a pilot study of the effect of manipulating 5-HT function. J Psychopharmacol 12: 155-160.

Aron AR, Fletcher PC, Bullmore ET, Sahakian BJ, Robbins TW (2003). Stop signal inhibition disrupted by damage to right inferior frontal gyrus in humans. Nat Neurosc 6: 115-116.

Attenburrow MJ, Mitter PR, Whale R, Terao T, Cowen PJ (2001). Low-dose citalopram as a 5-HT neuroendocrine probe. Psychopharmacology 155: 323-326.

Attenburrow MJ, Williams C, Odontiadis J, Reed A, Powell J, Cowen PJ et al (2003). Acute administration of nutritionally sourced tryptophan increases fear recognition. Psychopharmacology 169: 104-107.

Bechara A, Damasio AR, Damasio H, Anderson SW (1994). Insensitivity to future consequences following damage to human prefrontal cortex. Cognition 50: 7-15.

Bel N, Artigas F (1992). Fluvoxamine preferentially increases extracellular 5-hydroxytryptamine in the raphe nuclei: an in vivo microdialysis study. Eur J Pharmacol 229: 101-103.

Bell CJ, Nutt DJ (1998). Serotonin and panic. Br J Psychiatry 172: 465-471.

Blair RJ, Morris JS, Frith CD, Perrett DI, Dolan RJ (1999). Dissociable neural responses to facial expressions of sadness and anger. Brain 122: 883-893.

Casey BJ, Castellanos FX, Giedd JN, Marsh WL, Hamburger SD, Schubert $\mathrm{AB}$ et al (1997). Implication of right frontostriatal circuitry in response inhibition and attention-deficit/hyperactivity disorder. J Am Acad Child Adolesc Psychiatry 36: 374-383.

Coccaro EF, Kavoussi RJ, Hauger RL (1995). Physiological responses to $d$-fenfluramine and ipsapirone challenge correlate with indices of aggression in males with personality disorder. Int Clin Psychopharmacol 10: 177-179.

Darwin C (1872). The Expression of Emotions in Man and Animals. Philosophical Library: New York.

David DJ, Bourin M, Jego G, Przybylski C, Jolliet P, Gardier AM (2003). Effects of acute treatment with paroxetine, citalopram and venlafaxine in vivo on noradrenaline and serotonin outflow: a microdialysis study in Swiss mice. $\mathrm{Br} J$ Pharmacol 140: $1128-1136$.

Deakin JF (2003). Depression and antisocial personality disorder: two contrasting disorders of 5HT function. J Neural Transm 64(Suppl): 79-93.

Deakin JFW, Graeff FG (1991). 5-HT and mechanisms of defence. J Psychopharmacol 5: 305-315.

Dinn WM, Harris CL (2000). Neurocognitive function in antisocial personality disorder. Psychiatry Res 97: 173-190.

Dolan M, Anderson IM, Deakin JF (2001). Relationship between 5 -HT function and impulsivity and aggression in male offenders with personality disorders. Br J Psychiatry 178: 352-359.

Dolan M, Park I (2002). The neuropsychology of antisocial personality disorder. Psychol Med 32: 417-427.

Drevets WC, Frank E, Price JC, Kupfer DJ, Holt D, Greer PJ et al (1999). PET imaging of serotonin 1A receptor binding in depression. Biol Psychiatry 46: 1375-1387.

Ekman P, Friesen WV (1976). Pictures of Facial Affect. Consulting Psychologists: Palo Alto.

Elliott R, Dolan RJ, Frith CD (2000a). Dissociable functions in the medial and lateral orbitofrontal cortex: evidence from human neuroimaging studies. Cereb Cortex 10: 308-317.

Elliott R, Friston KJ, Dolan RJ (2000b). Dissociable neural responses in human reward systems. J Neurosci 20: 6159-6165.

Elliott R, Newman JL, Longe OA, Deakin JF (2003). Differential response patterns in the striatum and orbitofrontal cortex to financial reward in humans: a parametric functional magnetic resonance imaging study. J Neurosci 23: 303-307.
Felton TM, Kang TB, Hjorth S, Auerbach SB (2003). Effects of selective serotonin and serotonin/noradrenaline reuptake inhibitors on extracellular serotonin in rat diencephalon and frontal cortex. Naunyn Schmiedeberg's Arch Pharmacol 367: 297-305.

First MB, Spitzer RL, Gibbon M, Williams JBW (2002). Structured Clinical Interview for DSM-IV-TR Axis I Disorders, Research Version, Non-patient Edition. Biometrics Research, New York State Psychiatric Institute: (SCID-I/NP) New York.

Fischer H, Wright CI, Whalen PJ, McInerney SC, Shin LM, Rauch SL (2003). Brain habituation during repeated exposure to fearful and neutral faces: a functional MRI study. Brain Res Bull 59: 387-392.

Fu CH, Williams SC, Cleare AJ, Brammer MJ, Walsh ND, Kim J et al (2004). Attenuation of the neural response to sad faces in major depression by antidepressant treatment: a prospective, event-related functional magnetic resonance imaging study. Arch Gen Psychiatry 61: 877-889.

Garavan H, Ross TJ, Murphy K, Roche RA, Stein EA (2002). Dissociable executive functions in the dynamic control of behavior: inhibition, error detection, and correction. Neuroimage 17: 1820-1829.

Gartside SE, Umbers V, Hajos M, Sharp T (1995). Interaction between a selective 5-HT1A receptor antagonist and an SSRI in vivo: effects on 5-HT cell firing and extracellular 5-HT. $\mathrm{Br} J$ Pharmacol 115: 1064-1070.

Graeff FG (2002). On serotonin and experimental anxiety. Psychopharmacology 163: 467-476.

Gur RE, McGrath C, Chan RM, Schroeder L, Turner T, Turetsky BI et al (2002). An fMRI study of facial emotion processing in patients with schizophrenia. Am J Psychiatry 159: 1992-1999.

Hariri AR, Bookheimer SY, Mazziotta JC (2000). Modulating emotional responses: effects of a neocortical network on the limbic system. Neuroreport 11: 43-48.

Hariri AR, Tessitore A, Mattay VS, Fera F, Weinberger DR (2002). The amygdala response to emotional stimuli: a comparison of faces and scenes. Neuroimage 17: 317-323.

Harmer CJ, Bhagwagar Z, Perrett DI, Vollm BA, Cowen PJ, Goodwin GM (2003a). Acute SSRI administration affects the processing of social cues in healthy volunteers. Neuropsychopharmacology 28: 148-152.

Harmer CJ, Rogers RD, Tunbridge E, Cowen PJ, Goodwin GM (2003b). Tryptophan depletion decreases the recognition of fear in female volunteers. Psychopharmacology 167: 411-417.

Harmer CJ, Shelley NC, Cowen PJ, Goodwin GM (2004). Increased positive versus negative affective perception and memory in healthy volunteers following selective serotonin and norepinephrine reuptake inhibition. Am J Psychiatry 161: 1256-1263.

Haxby JV, Hoffman EA, Gobbini MI (2002). Human neural systems for face recognition and social communication. Biol Psychiatry 51: 59-67.

Hester R, Fassbender C, Garavan H (2004). Individual differences in error processing: a review and reanalysis of three eventrelated fMRI studies using the Go/No-go task. Cereb Cortex 14: 986-994.

Hetem LA, de Souza CJ, Guimaraes ES, Zuardi AW, Graeff FG (1996). Effect of d-fenfluramine on human experimental anxiety. Psychopharmacology 127: 276-282.

Horn NR, Dolan M, Elliott R, Deakin JF, Woodruff PW (2003). Response inhibition and impulsivity: an fMRI study. Neuropsychologia 41: 1959-1966.

Jetty PV, Charney DS, Goddard AW (2001). Neurobiology of generalized anxiety disorder. Psychiatr Clin N Am 24: 75-97.

Karnath HO (2001). New insights into the functions of the superior temporal cortex. Nat Rev Neurosci 2: 568-576.

Kawashima R, Satoh K, Itoh H, Ono S, Furumoto S, Gotoh R et al (1996). Functional anatomy of GO/NO-GO discrimination and response selection - a PET study in man. Brain Res 728: 79-89. 
Keightley ML, Winocur G, Graham SJ, Mayberg HS, Hevenor SJ, Grady CL (2003). An fMRI study investigating cognitive modulation of brain regions associated with emotional processing of visual stimuli. Neuropsychologia 41: 585-596.

Kringelbach ML, Rolls ET (2004). The functional neuroanatomy of the human orbitofrontal cortex: evidence from neuroimaging and neuropsychology. Prog Neurobiol 72: 341-372.

Lange K, Williams LM, Young AW, Bullmore ET, Brammer MJ, Williams SC et al (2003). Task instructions modulate neural responses to fearful facial expressions. Biol Psychiatry 53: 226-232.

Lawrence NS, Williams AM, Surguladze S, Giampietro V, Brammer MJ, Andrew C et al (2004). Subcortical and ventral prefrontal cortical neural responses to facial expressions distinguish patients with bipolar disorder and major depression. Biol Psychiatry 55: 578-587.

Liddle PF, Kiehl KA, Smith AM (2001). Event-related fMRI study of response inhibition. Hum Brain Mapp 12: 100-109.

Longe O, Deakin JFW, Elliott R (2003). Anticipating reinforcement in Unipolar Depression Presented at the ninth International Conference on Functional Mapping of the Human Brain, June 19-22, 2003, New York, NY. Available on CD-Rom in NeuroImage 19 (Volume 2).

Mann JJ, Brent DA, Arango V (2001). The neurobiology and genetics of suicide and attempted suicide: a focus on the serotonergic system. Neuropsychopharmacology 24: 467-477.

Mann JJ, McBride PA, Malone KM, DeMeo M, Keilp J (1995). Blunted serotonergic responsivity in depressed inpatients. Neuropsychopharmacology 13: 53-64.

Menon V, Adleman NE, White CD, Glover GH, Reiss AL (2001). Error-related brain activation during a Go/NoGo response inhibition task. Hum Brain Mapp 12: 131-143.

Miller HE, Deakin JF, Anderson IM (2000). Effect of acute tryptophan depletion on $\mathrm{CO}_{2}$-induced anxiety in patients with panic disorder and normal volunteers. Br J Psychiatry 176: 182-188.

Moret C, Briley M (1996). Effects of acute and repeated administration of citalopram on extracellular levels of serotonin in rat brain. Eur J Pharmacol 295: 189-197.

Morris JS, Buchel C, Dolan RJ (2001). Parallel neural responses in amygdala subregions and sensory cortex during implicit fear conditioning. Neuroimage 13: 1044-1052.

Morris JS, Frith CD, Perrett DI, Rowland D, Young AW, Calder AJ et al (1996). A differential neural response in the human amygdala to fearful and happy facial expressions. Nature 383: 812-815

Mortimore C, Anderson IM (2000). d-Fenfluramine in panic disorder: a dual role for 5-hydroxytryptamine. Psychopharmacology 149: 251-258.

Mostofsky SH, Schafer JG, Abrams MT, Goldberg MC, Flower AA, Boyce A et al (2003). fMRI evidence that the neural basis of response inhibition is task-dependent. Brain Res Cogn Brain Res 17: 419-430.

Nemeroff CB (2002). Recent advances in the neurobiology of depression. Psychopharmacol Bull 36(Suppl 2): 6-23.

O’Doherty J, Kringelbach ML, Rolls ET, Hornak J, Andrews C (2001). Abstract reward and punishment representations in the human orbitofrontal cortex. Nat Neurosci 4: 95-102.

O'Keane V, McLoughlin D, Dinan TG (1992). D-Fenfluramineinduced prolactin and cortisol release in major depression: response to treatment. J Affect Disord 26: 143-150.

Phan KL, Wager T, Taylor SF, Liberzon I (2002). Functional neuroanatomy of emotion: a meta-analysis of emotion activation studies in PET and fMRI. Neuroimage 16: 331-348.

Phillips ML, Medford N, Young AW, Williams L, Williams SC, Bullmore ET et al (2001). Time courses of left and right amygdalar responses to fearful facial expressions. Hum Brain Mapp 12: 193-202.
Phillips ML, Young AW, Senior C, Brammer M, Andrew C, Calder AJ et al (1997). A specific neural substrate for perceiving facial expressions of disgust. Nature 389: 495-498.

Rogers RD, Tunbridge EM, Bhagwagar Z, Drevets WC, Sahakian BJ, Carter CS (2003). Tryptophan depletion alters the decisionmaking of healthy volunteers through altered processing of reward cues. Neuropsychopharmacology 28: 153-162.

Rubia K, Smith AB, Brammer MJ, Taylor E (2003). Right inferior prefrontal cortex mediates response inhibition while mesial prefrontal cortex is responsible for error detection. Neuroimage 20: 351-358.

Schwartz CE, Wright CI, Shin LM, Kagan J, Whalen PJ, McMullin KG et al (2003). Differential amygdalar response to novel versus newly familiar neutral faces: a functional MRI probe developed for studying inhibited temperament. Biol Psychiatry 53: 854-862.

Sheehan DV, Lecrubier Y, Sheehan KH, Amorim P, Janavs J, Weiller E et al (1998). The Mini-International Neuropsychiatric Interview (MINI): the development and validation of a structured diagnostic psychiatric interview for DSM-IV and ICD-10. J Clin Psychiatry 59(Suppl 20): 22-33.

Sheline YI, Barch DM, Donnelly JM, Ollinger JM, Snyder AZ, Mintun MA (2001). Increased amygdala response to masked emotional faces in depressed subjects resolves with antidepressant treatment: an fMRI study. Biol Psychiatry 50: 651-658.

Sprengelmeyer R, Rausch M, Eysel UT, Przuntek H (1998). Neural structures associated with recognition of facial expressions of basic emotions. Proc R Soc Lond Ser B 265: 1927-1931.

Stockmeier CA (2003). Involvement of serotonin in depression: evidence from postmortem and imaging studies of serotonin receptors and the serotonin transporter. J Psychiatr Res 37: 357-373.

Surguladze SA, Brammer MJ, Young AW, Andrew C, Travis MJ, Williams SC et al (2003). A preferential increase in the extrastriate response to signals of danger. Neuroimage 19: $1317-1328$.

Talairach J, Tournoux P (1988). Coplanar Stereotactic Atlas of the Human Brain. George Thieme Verlag: Stuttgart.

Thomas KM, Drevets WC, Dahl RE, Ryan ND, Birmaher B, Eccard $\mathrm{CH}$ et al (2001). Amygdala response to fearful faces in anxious and depressed children. Arch Gen Psychiatry 58: 1057-1063.

Völlm B, Richardson P, Stirling J, Elliott R, Dolan M, Chaudhry I et al (2004). Neurobiological substrates of antisocial and borderline personality disorder: preliminary results of a functional fMRI study. Crim Behav Ment Health 14: 39-54.

Wallis JD, Dias R, Robbins TW, Roberts AC (2001). Dissociable contributions of the orbitofrontal and lateral prefrontal cortex of the marmoset to performance on a detour reaching task. Eur J Neurosci 13: 1797-1808.

Whalen PJ, Shin LM, McInerney SC, Fischer H, Wright CI, Rauch SL (2001). A functional MRI study of human amygdala responses to facial expressions of fear versus anger. Emotion 1: 70-83.

Winston JS, O'Doherty J, Dolan RJ (2003). Common and distinct neural responses during direct and incidental processing of multiple facial emotions. Neuroimage 20: 84-97.

Worsley KJ, Marrett S, Neelin P, Vandal AC, Friston KJ, Evans AC (1996). A unified statistical approach for determining significant signals in images of cerebral activation. Hum Brain Mapp 4: 58-73.

Yang TT, Menon V, Eliez S, Blasey C, White CD, Reid AJ et al (2002). Amygdalar activation associated with positive and negative facial expressions. Neuroreport 13: 1737-1741.

Zald DH, Kim SW (2001). The orbitofrontal cortex. In: Salloway SP, Malloy PF, Duffy JD (eds). The Frontal Lobes and Neuropsychiatric Illness. APPI: Washington DC, London, England. pp 33-69. 